\title{
El aula de música como ambiente sonoro de aprendizaje en Educación Infantil
}

The music classroom as a sound environment for learning in early childhood education

\author{
Marta Ciurana González \\ martaciuranagonzalez@gmail.com \\ ORCID: http://0000-0001-6625-1097 \\ Miquel Alsina Tarrés \\ miquel.alsina@udg.edu \\ Departamento de Didácticas Específicas \\ Universidad de Girona \\ Gerona, España \\ ORCID: http://0000-0003-4599-6852
}

doi: 10.7203/LEEME.44.15595

Recibido: 12-07-2019 Aceptado: 31-10-2019. Contacto y correspondencia: Miquel Alsina Tarrés, Departamento de Didácticas Específicas Universidad de Girona, Plaza Sant Domènech 9, 17004. Gerona. España.

\section{Resumen}

Se presenta una investigación de campo que parte de la creación de un ambiente sonoro de aprendizaje en una escuela de Educación Infantil y Primaria. En este entorno, a través del juego libre, se observa y analiza la interacción con el medio del educando de segundo ciclo de Educación Infantil (n=104), organizados en 10 grupos de perfil homogéneo. En segundo lugar, se comparan los resultados entre las diferentes edades de la etapa educativa. Se sigue una metodología de investigación mixta, cualitativa y cuantitativa, bajo el paradigma del diseño secuencial exploratorio. Las sesiones se grabaron en vídeo y se analizaron con una tabla de observación individual (TOIM) basándose en una categorización de los resultados preliminares. Los resultados muestran como el nuevo entorno y los objetos ofrecidos han permitido al alumnado desarrollar aprendizajes de manera global, más allá de aspectos relacionados con la exploración sonora. Se observan así mismo diferencias, de menos a más, en cómo se desarrollan estudiantes de diferentes edades, especialmente en referencia al tipo de juego, la aproximación a los objetos, la acción de producir y escuchar el sonido y en las relaciones sociales que se establecen.

Palabras clave: Exploración sonora, espacio de aprendizaje, juego libre, aprendizaje globalizado, Educación Infantil.

Abstract

A field investigation is presented that starts from the creation of a sound learning environment in pre-school and primary education. In this environment, through free play, the interaction with the students' environment in the second cycle of early childhood education $(\mathrm{n}=104)$, organized in 10 homogeneous groups, is observed and analyzed. Secondly, the results are compared between the different ages of the sample. We follow a methodology of mixed research, qualitative and quantitative, under the paradigm of exploratory sequential design. The sessions were recorded on video and analyzed with an individual observation table (TOIM) based on a categorization of the preliminary results. The results show how the new space and their objects allow students to develop learning in a global manner, beyond aspects related to sound exploration. Differences are also observed, from less to more, in the way of how behavior from students of different ages differs, especially in reference to the type of game, the approach to objects, the action of producing and listening to sound and in the social relations that are established.

Key words: Sound exploration, learning environments, aesthetic, free play, global learning, Pre-school Education.

@MARTA CIURANA GONZÁLEZZ Y MIQUEL ALSINA TARRÉS. THE CONTENT OF THIS ARTICLE IS THE SOLE RESPONSIBILITY OF THE AUTHORS. THE REVISTA ELECTRÓNICA DE LEEME AND UNIVERSITAT DE VALĖNCIA ARE NOT LIABLE FOR ANY LEGAL ACTIONS THAT MAY ARISE INVOLVING THE ARTICLE'S CONTENT. REVISTA ELECTRÓNICA DE LEEME -LISTA ELECTRÓNICA EUROPEA DE MÚSICA EN LA EDUCACIÓN-HTTP:// /OJS.UV.ES/INDEX/PHP/LEEME/INDEX. ISSN: 1575-9563. EDITORES: UNIVERSIDAD DE VALENCIA Y JESÚS TEJADA GIMÉNEZ. VISIBILIDAD DE ESTA REVISTA: SCOPUS, EMERGING SOURCES CITATION INDEX (CLARIVATE), EBSCO, CINDOC (CSIC), CITEFACTOR, COPAC, DIALNET, DICE (CSIC), DOAJ, E-REVISTAS
(CSIC), EBSCO PREMIER, ERIH+, GALE CENGAGE LEARNING, IN-RECS, IRESIE, LATINDEX, MIAR, OCLC WORLDCAT, RESH, REDIB, RILM CORE JOURNALS, SUDOC, ULRICHS, ESTA REVISTA ESTÁ PUBLICADA CON EL APOYO INSTITUCIONAL DE REDIRIS-CONSEJO SUPERIOR DE INVESTIGACIONES CIENTIFICAS Y ES DE ACCESO LIBRE. CREATIVE COMMONS LICENSE 4.O BY 


\section{Introducción}

La música, como elemento cultural y comunicativo, configura un rasgo humano universal presente desde la primera infancia (Blaking, 1973; Peretz, 2006). Del mismo modo como ocurre en otras capacidades, cuanto antes se promueva una experiencia activa en la vida musical del niño o de la niña mayor será el impacto sobre su posterior maduración (Levinowitz, 1998). Los elementos esenciales en el planteamiento de un programa musical de calidad para los pequeños deberían promover experiencias positivas, como el logro, la satisfacción y el disfrute, a través de la participación sensorial inclusiva (Tammerman, 2000; Thompson, 2003; Hallam, 2010). Por otro lado, la música tiene una fuerte influencia en la plasticidad del cerebro, de modo que hay una creciente evidencia sobre el nivel de trasferencia positiva de la experiencia musical a los dominios no musicales, como, por ejemplo, la motricidad, la gestión emocional y las habilidades sociales comunicativas (Jäncke, 2009; Ilari, 2016).

Entre los dos y los seis años, la capacidad de percibir y sentir alcanza su máximo potencial (Calvo y Bernal, 2000). En estas edades, los chicos y las chicas están siempre aprendiendo, y la mayor parte de la conducta la asimilan de forma inconsciente, a través de la acción y de la organización de sus percepciones. Esta primera etapa de aprendizajes musicales debe revalorizarse y entenderse como significativa en la evolución escolar posterior del alumno (Zur y Johnson-Green, 2008).

\subsection{La música en el segundo ciclo de Educación Infantil}

Al inicio del segundo ciclo, y a lo largo de la etapa, el alumnado de Educación Infantil desarrolla, entre o tras, su dimensión social y sus capacidades de interacción con el entorno. En referencia a la música, al infante le atrae y le gusta el sonido y participar de actividades donde la expresión musical tenga un papel protagonista. Desarrolla el placer por la exploración de instrumentos musicales y todo tipo de objetos susceptibles de producir sonido, así como sus gustos y preferencias musicales en cuanto a repertorio y actividades musicales en el aula (Delalande y Cornara, 2010; Pérez-Moreno y Reverté, 2019).

A continuación, se plasman algunas peculiaridades genéricas en la maduración musical del discente para cada una de las edades del segundo ciclo de Infantil, centradas principalmente en la expresión y la percepción (Calvo y Bernal, 2000; Pascual, 2006):

- A los 3 años, le gusta experimentar con grupos rítmicos y puede reproducir estructuras rítmicas de forma regular y monótona. Puede imitar sonidos y agrupar entre sí elementos sonoros. Está descubriendo las posibilidades rítmico-musicales de las palabras y puede reproducir canciones con gran contenido onomatopéyico. Reconoce melodías simples y le gusta experimentar con instrumentos de percusión. 


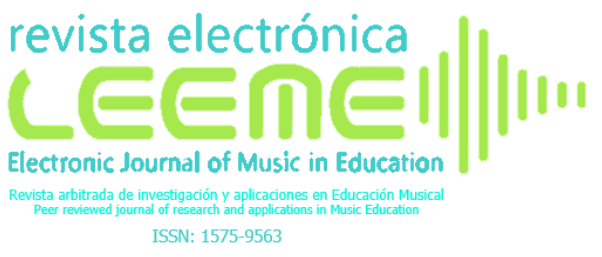

SECCIÓN ARTICULOS: MARTA CIURANA GONZÁLEZ Y MIQUEL ALSINA TARRÉS JUEGO Y SONIDO EN EDUCACIÓN INFANTIL: EL AULA DE MÚSICA COMO ESPACIO SONORO DE (REVISTA ELECTRÓNICA DE LEEME) NÚMERO 44, PP. 42-62 HTTPS://OJS.UVIES/INDEX.PPP/LEEME/INDEX

- A los 4 años, disfruta con las relaciones sociales en las que interviene la música. Hay un desarrollo notable de la creatividad. Juega a juegos simples acompañándolos de una canción, dramatizando lo que canta. Un grupo de estudiantes es capaz de llevar el mismo ritmo, y aumenta su memoria auditiva. Le gusta explorar objetos sonoros.

- A los 5 años, se produce una evolución sustancial en la conducta musical, sobre todo en relación con el aspecto rítmico. Sincroniza los movimientos con la música y es capaz de crear canciones muy sencillas con pocos sonidos. Disfruta jugando con ejercicios de concentración rítmica, sonora, etc. Puede ordenar y clasificar sonidos, y también explorar otros instrumentos.

En referencia a esta etapa educativa, el documento curricular competente nos recuerda que la adquisición progresiva del lenguaje musical se asemeja a la de una lengua (Generalidad de Catalunya, 2016). En este sentido, el currículum incita a la comunidad de educadores a privilegiar el sonido como materia primera del arte musical, y a hacerlo desde una perspectiva amplia e inclusiva:

"Desde las vivencias y el conocimiento que recibimos a partir del sonido que nos rodea; desde los vínculos que creamos con las personas que interaccionan sonoramente con nosotros; y desde los referentes musicales que escuchamos y de la manera cómo vivimos las experiencias musicales en nuestras vidas" (p.24).

El currículum de la etapa recuerda, a su vez, cómo en la infancia la curiosidad por el mundo sonoro es natural y, a su vez, refinada. Y se insiste en su valor educativo afirmando que:

“Conocer y reconocer sonidos, compararlos, relacionarlos, clasificarlos, adjetivarlos... Ofrece a los niños la possibilidad de asimilar una información valiosa de conocimiento del mundo, además de despertarles e incitarlos al gusto por el sonido, condición indispensable para disfrutar de la música" (p.25).

\subsection{La exploración sonora en la etapa 3-6}

El sonido es la materia constituyente e indispensable para que la música exista. El gusto por el sonido es muy vivo y natural desde la primera infancia, en la que el infante escucha y explora de forma intuitiva y espontánea. Igualmente, su relación con el mundo sonoro es intensa, y desde edades tempranas mantienen una relación lúdica con el sonido, guiada por la curiosidad y el instinto de exploración (Kemple, Batey y Hartle, 2004).

Delalande, desde una base constructivista, argumenta que es preferible despertar el interés por aprender antes que enseñar a hacer las cosas, dando protagonismo al instinto y a la propia iniciativa. Se trata de acompañar en el proceso de descubrir y alentando comportamientos

@MARTA CIURANA GONZÁLEZ Y MIQUEL ALSINA TARRÉS. THE CONTENT OF THIS ARTICLE IS THE SOLE RESPONSIBILITY OF THE AUTHORS. THE REVISTA ELECTRÓNICA DE LEEME AND UNIVERSITAT DE VALĖNCIA ARE NOT LIABLE FOR ANY LEGAL ACTIONS THAT MAY ARISE INVOLVING THE ARTICLE'S CONTENT REVISTA ELECTRÓNICA DE LEEME —LISTA ELECTRÓNICA EUROPEA DE MÚSICA EN LA EDUCACIÓN-HTTP://OJS.UV.ES/INDEX/PHP/LEEME/INDEX. ISSN: 1575-9563. EDITORES: UNIVERSIDAD DE VALENCIA Y JESÚS TEJADA GIMÉNEZ. VISIBILIDAD DE ESTA REVISTA: SCOPUS, EMERGING SOURCES CITATION INDEX (CLARIVATE), EBSCO, CINDOC (CSIC), CITEFACTOR, COPAC, DIALNET, DICE (CSIC), DOAJ, E-REVISTAS (CSIC), EBSCO PREMIER, ERIH+, GALE CENGAGE LEARNING, IN-RECS, IRESIE, LATINDEX, MIAR, OCLC WORLDCAT, RESH, REDIB, RILM CORE JOURNALS, SUDOC, ULRICHS, ESTA REVISTA ESTÁ PUBLICADA CON EL APOYO INSTITUCIONAL DE REDIRIS-CONSEJO SUPERIOR DE INVESTIGACIONES CIENTIFICAS Y ES DE ACCESO LIBRE. CREATIVE COMMONS LICENSE 4.O BY 
espontáneos guiándolos suficientemente (Delalande, 1995). El mismo autor expone cómo primero el educando explora el objeto material que produce el sonido, interesándose por su mecanismo. En este caso, la motricidad es la principal protagonista, por delante de la propia escucha. En un segundo momento, el discente explora el objeto sonoro y se concentra en el sonido que produce, conduciéndole así a "la búsqueda de la idea musical". Y, a continuación, ya en una fase más avanzada, es capaz de centrarse en una idea y desarrollarla (Delalande, 2013, p.155).

\subsection{Espacio, ambientes de aprendizaje y juego}

En la literatura educativa a menudo se utilizan los conceptos de espacio y ambiente como términos equivalentes. De forma genérica, lo que caracteriza el espacio es su propia condición como elemento físico, pero también como elemento más allá de lo físico, puesto que el espacio se configura como un elemento educativo en sí mismo, como un instrumento pedagógico de primer orden y un componente didáctico primordial (Gairín, 1995). En verdad, es posible diferenciar conceptualmente el espacio del ambiente. Mientras que el primer término se refiere primordialmente a los objetos materiales, el segundo aglutina muy diversas cualidades y resulta por tanto más adecuado a la hora de articular el pensamiento y la acción educativa. Iglesias (2008) propone distinguir cuatro dimensiones en la definición, observación o evaluación de todo ambiente educativo: física, funcional, temporal y relacional.

En la línea de lo expuesto, hay que tener en cuenta que "la disposición del ambiente influye de forma significativa en aquellos que lo ocupan" (Cabello, 2011, p.196). En consecuencia, como toda acción educativa en su conjunto, la acción de pensar y crear un ambiente de aprendizaje requiere una planificación cuidadosa y el espacio como elemento facilitador de valores transversales como la inclusión y la sostenibilidad (Laorden y Pérez, 2002; Saenz-Rico, Benítez, Neira, Sobrino y D’angelo, 2015).

Nos interesamos también, a la hora de pensar el espacio como un componente atractivo y la vez pedagógico, en aspectos conceptuales relacionados con las instalaciones artísticas, las cuales Abad y Ruiz de Velasco (2014) definen en su propuesta como "espacios concebidos a partir del encuentro entre una propuesta estética y una fundamentación pedagógica inspirada en la práctica psicomotriz Aucouturier. Este diálogo posibilita y favorece el juego pre-simbólico y simbólico en un contexto relacional” (p.11).

Un ambiente de aprendizaje debe facilitar y sugerir diversas posibilidades de acción. Debe permitir entrar en juego al infante, por lo tanto, una condición indispensable es ofrecer espacios flexibles y transformables. En el espacio, se presentan objetos y materiales de forma ordenada para que el alumnado entienda que existe una situación inicial; de este modo, puede apropiarse e interpretar el espacio, hacerlo propio, elaborando así nuevos significados. Por otra parte, hay que generar unos ambientes de aprendizaje bellos y atractivos. Tal y como remarca Trueba (2015,

@MARTA CIURANA GONZÁLEZY MIQUEL ALSINA TARRÉS. THE CONTENT OF THIS ARTICLE IS THE SOLE RESPONSIBILITY OF THE AUTHORS. THE REVISTA ELECTRÓNICA DE LEEME AND UNIVERSITAT DE VALÈNCIA ARE NOT LIABLE FOR ANY LEGAL ACTIONS THAT MAY ARISE INVOLVING THE ARTICLE'S CONTENT. REVISTA ELECTRÓNICA DE LEEME —LISTA ELECTRÓNICA EUROPEA DE MÚSICA EN LA EDUCACIÓN-HTTP://OJS.UV.ES/INDEX/PHP/LEEME/INDEX. ISSN: 1575-9563. EDITORES: UNIVERSIDAD DE VALENCIA Y JESÚS TEJADA GIMÉNEZ. VISIBILIDAD DE ESTA REVISTA: SCOPUS, EMERGING SOURCES CITATION INDEX (CLARIVATE), EBSCO, CINDOC (CSIC), CITEFACTOR, COPAC, DIALNET, DICE (CSIC), DOAJ, E-REVISTAS (CSIC), EBSCO PREMIER, ERIH+, GALE CENGAGE LEARNING, IN-RECS, IRESIE, LATINDEX, MIAR, OCLC WORLDCAT, RESH, REDIB, RILM CORE JOURNALS, SUDOC, ULRICHS, ESTA REVISTA ESTÁ PUBLICADA CON EL APOYO INSTITUCIONAL DE REDIRIS-CONSEJO SUPERIOR DE INVESTIGACIONES CIENTIFICAS Y ES DE ACCESO LIBRE. CREATIVE COMMONS LICENSE 4.O BY 
p.125), "sabemos que los niños aprecian, recrean, disfrutan y se emocionan con lo auténticamente hermoso".

Silvente (2017) nos da algunos consejos a la hora de construir ambientes de aprendizaje. Por ejemplo, en primer lugar, buscar la calidad y huir de la masificación. Para conseguirlo, es preferible que predominen los objetos abiertos, que ofrecen múltiples usos y favorecen el pensamiento creativo y divergente. En segundo lugar, es necesaria una vinculación entre las partes y, a su vez, una limitación clara entre los espacios. Finalmente, debería permitirse al alumnado una circulación libre por el aula.

Según Torelli y Durrett (1996), otro aspecto importante a la hora de intervenir en el espacio, creando ambientes de aprendizaje de calidad, es que estos por si mismos generen autoaprendizaje, favoreciendo la emergencia de nuevos interrogantes cognitivos y a su vez desarrollando los ya conocidos. También es positivo conseguir que sean lugares emocionales, promoviendo las relaciones y la comunicación entre los participantes, permitiéndoles disfrutar también de momentos de intimidad y quietud (Young, 2003; Young y Gillen, 2007).

Una forma espontánea e inclusiva de interacción entre los más pequeños y su entorno es el juego libre. El juego libre es importante para que el infante encuentre un equilibrio entre la realidad y la subjetividad, el juego le permite ser otra persona con seguridad. A lo largo de la infancia, y gracias especialmente al recurso del juego, la percepción de la realidad evoluciona hacia una consciencia más realista (Abad, 2008). El juego simbólico es el más representativo en la Educación Infantil, en este el alumno despliega su pequeño mundo interior y acepta los conocimientos de los demás. Aun así, cuando aparece el juego simbólico este convive con los juegos motores o motrices, que no desaparecen. Más aún, es necesario programarlos conscientemente y que dispongan de un espacio y un tiempo concreto (Romero y Gómez, 2011; Díez, 2013).

\section{Método}

\subsection{Paradigma y preguntas de investigación}

Se ha llevado a cabo una investigación de campo, cuyo paradigma se encuadra en el modelo cuasi experimental, de tipo exploratorio-descriptivo (Río Sadornil, 2003). Con este fin, se ha concebido y transformado el aula de música, en la cual se ha introducido y observado una muestra determinada (Creswell y Creswell, 2018). En este diseño, la variable introducida ha sido la adecuación material y el uso del espacio para la creación del ambiente sonoro. Este medio, adaptado y transformado ad hoc para la ocasión, es entendido como objeto educativo de experimentación, bajo un concepto exploratorio y lúdico. 
Nuestro papel como investigadores ha sido el de concebir, construir e instalar un completo elenco de objetos para posteriormente observar, categorizar y evaluar los comportamientos y las interacciones con el medio. La finalidad es la creación de un cuerpo teórico o conjunto de hipótesis de trabajo (Young, 2016).

Para la obtención y el análisis de datos se ha partido de un procedimiento mixto, cualitativo y cuantitativo, que se acomoda al paradigma exploratorio, con diseño secuencial (Leavy, 2017). En una primera fase, se ha utilizado una metodología cualitativa a partir de la observación naturalista no participante. Para ello, en aras también de la validez interna del diseño, se construyó un grupo de enfoque de 12 estudiantes (una pareja de cada grupo clase) que, a modo de prueba piloto, experimentó e interactuó durante 30 minutos en el espacio. Como aconseja la literatura, estos discentes quedaron excluidos en la extracción final de resultados. A su vez, este preliminar permitió repensar alguno de los objetos, la organización del espacio y, principalmente, obtener evidencias sobre la exploración e interacciones que se producían en el medio. También en esta fase, se establecieron las cuatro categorías de análisis, para lo que se tuvo también en cuenta el desglose en dimensiones propuesto por Iglesias (2008). Entendemos que las dimensiones física y temporal las fijamos los investigadores y las dos restantes (funcional y relacional) son el objeto de la investigación.

- Categoría 1. Uso sonoro y/o musical (dimensión funcional): referida a la exploración sonora de los objetos, la apreciación y experimentación de las cualidades acústicas del sonido producido, es decir, la altura, la intensidad, la duración y el timbre. Incluye también la observación de procesos facilitadores en los cuales, a través del material facilitado, aparezcan expresiones vocales, canciones o danzas.

- Categoría 2. Relación entre participantes (dimensión relacional): centrada en la dimensión comunicativa de la actividad, como el registro de expresiones verbales, emisión de juicios, valoraciones, y cualquier elemento que indique interacción entre dos o más estudiantes. También incluye la forma de agrupación entorno a las actividades, si es individual, en parejas, etc.

- Categoría 3. Interacción con el material (dimensión funcional): dedicada al registro de acciones y actitudes en relación con los objetos, las formas de manipularlos, de clasificarlos y de recolocarlos en el espacio. Es una categoría abierta a su vez al registro de acciones inesperadas producto de la creación y fantasía del alumnado, y a la recogida de interrogantes y conflictos cognitivos.

- Categoría 4. Interés y motivación (dimensión relacional): recoge las percepciones sobre el interés por el ambiente sonoro y la valoración de la propuesta a través de las expresiones verbales y corporales. Así como la atención y dedicación a la actividad desde el inicio y a lo largo de todo el tiempo dedicado.

@MARTA CIURANA GONZÁLEZY MIQUEL ALSINA TARRÉS. THE CONTENT OF THIS ARTICLE IS THE SOLE RESPONSIBILITY OF THE AUTHORS. THE REVISTA ELECTRÓNICA DE LEEME AND UNIVERSITAT DE VALENCIA ARE NOT LIABLE FOR ANY LEGAL ACTIONS THAT MAY ARISE INVOLVING THE ARTICLE'S CONTENT REVISTA ELECTRÓNICA DE LEEME -LISTA ELECTRÓNICA EUROPEA DE MÚSICA EN LA EDUCACIÓN-HTTP://OJS.UV.ES/INDEX/PHP/LEEME/INDEX. ISSN: 1575-9563. EDITORES: UNIVERSIDAD DE VALENCIA Y JESÚS TEJADA GIMÉNEZ. VISIBILIDAD DE ESTA REVISTA: SCOPUS, EMERGING SOURCES CITATION INDEX (CLARIVATE), EBSCO, CINDOC (CSIC), CITEFACTOR, COPAC, DIALNET, DICE (CSIC), DOAJ, E-REVISTAS (CSIC), EBSCO PREMIER, ERIH+, GALE CENGAGE LEARNING, IN-RECS, IRESIE, LATINDEX, MIAR, OCLC WORLDCAT, RESH, REDIB, RILM CORE JOURNALS, SUDOC, ULRICHS, ESTA REVISTA ESTÁ PUBLICADA CON EL APOYO INSTITUCIONAL DE REDIRIS-CONSEJO SUPERIOR DE INVESTIGACIONES CIENTIFICAS Y ES DE ACCESO LIBRE. CREATIVE COMMONS LICENSE 4.O BY 


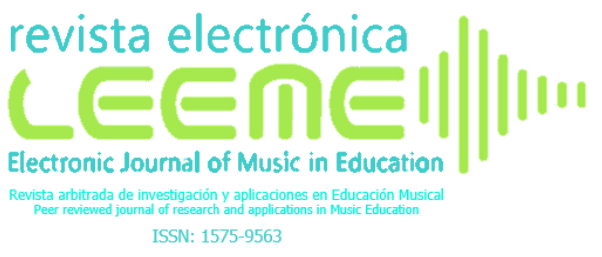

SECCIÓN ARTICULOS: MARTA CIURANA GONZÁLEZ Y MIQUEL ALSINA TARRÉS JUEGO Y SONIDO EN EDUCACIÓN INFANTIL: EL AULA DE MÚSICA COMO ESPACIO SONORO DE (REVISTA ELECTRÓNICA DE LEEME) NÚMERO 44, PP. 42-62 HTTPS://OJS.UVIES/INDEX.PHP/LEEME/INDEX

En la segunda fase, se mantiene el enfoque cualitativo de la investigación, pero se suma una herramienta cuantitativa complementaria. Esta consistente en una tabla de observación para medir los resultados en las cuatro categorías tras el visionado en vídeo de todas las sesiones. Cada uno de los videos se ha visionado diversas veces hasta rellenar una tabla individual de observación de la interacción con el medio (TOIM), aplicada a cada alumno participante. De este modo, al final del proceso, se obtuvo un registro para cada alumno con cada una de las cuatro categorías valorada sobre la escala: nada, poco, bastante o mucho. El registro cualitativo se mantiene, de modo que cada ficha individual incluye un apartado de observaciones focalizadas sobre los mismos cuatro apartados.

El objetivo de la investigación es dar respuesta a estas dos preguntas:

- ¿Cómo interacciona con el medio, a través del juego libre, el alumnado de segundo ciclo de Educación Infantil en un ambiente sonoro de aprendizaje?

- ¿Qué evolución se observa en los resultados entre estudiantes de segundo ciclo de Educación Infantil?

Señalar, por último, que las familias y el centro autorizaron el protocolo y uso de los datos obtenidos, conociendo previamente el código ético de la investigación.

\subsection{Contextualización y características de la muestra}

Esta investigación se ha llevado a cabo en una escuela pública de Educación Infantil y Primaria de la provincia de Gerona, que atiende cerca de 400 estudiantes. Sus familias son, en un $100 \%$, de origen extranjero. Entre ellos, predomina un número mayoritario de familias originarias de Marruecos y Gambia, seguidas por las de Senegal, Mali, y Honduras, entre otros. En total hay familias de hasta 17 países diferentes. La escuela está situada en una zona dónde, aproximadamente, más de un $40 \%$ de la población es de origen extranjero, aunque hay muchos niños y jóvenes de estas familias que ya han nacido en España.

La muestra la constituyó un total de 104 estudiantes (48 niños y 56 niñas) de seis aulas diferentes, de P3 hasta P5. Por consiguiente, las edades de los participantes estaban comprendidas entre los 3 y 5 años. Por el espacio del ambiente sonoro de aprendizaje pasaron una única sesión de 45 minutos, un total de 10 grupos de estudiantes, creados ad hoc con una composición de 8 a 14 participantes en cada uno. La creación de estos grupos se realizó con criterios de homogeneidad, respetando la adscripción al grupo clase natural de procedencia. Las sesiones se estructuraron en tres fases: presentación ( $5 \mathrm{~min}$.), juego libre (30 min.), recogida y cierre (10 min.).

Tabla 1. Grupos clase originales y grupos muestra de la investigación

\begin{tabular}{cccccc}
\hline Aula & Grupos muestra & Edad & $\mathbf{N}^{\circ}$ estudiantes & $\mathbf{N}^{\circ}$ niños & $\mathbf{N}^{0}$ niñas \\
\hline
\end{tabular}

@MARTA CIURANA GONZÁLEZY MIQUEL ALSINA TARRÉS. THE CONTENT OF THIS ARTICLE IS THE SOLE RESPONSIBILITY OF THE AUTHORS. THE REVISTA ELECTRÓNICA DE LEEME AND UNIVERSITAT DE VALENNCIA ARE NOT LIABLE FOR ANY LEGAL ACTIONS THAT MAY ARISE INVOLVING THE ARTICLE'S CONTENT. REVISTA ELECTRÓNICA DE LEEME —LISTA ELECTRÓNICA EUROPEA DE MÚSICA EN LA EDUCACIÓN-HTTP://OJS.UV.ES/INDEX/PHP/LEEME/INDEX. ISSN: 1575-9563. EDITORES: UNIVERSIDAD DE VALENCIA Y JESÚS TEJADA GIMÉNEZ. VISIBILIDAD DE ESTA REVISTA: SCOPUS, EMERGING SOURCES CITATION INDEX (CLARIVATE), EBSCO, CINDOC (CSIC), CITEFACTOR, COPAC, DIALNET, DICE (CSIC), DOAJ, E-REVISTAS (CSIC), EBSCO PREMIER, ERIH+, GALE CENGAGE LEARNING, IN-RECS, IRESIE, LATINDEX, MIAR, OCLC WORLDCAT, RESH, REDIB, RILM CORE JOURNALS, SUDOC, ULRICHS, ESTA REVISTA ESTÁ PUBLICADA CON EL APOYO INSTITUCIONAL DE REDIRIS-CONSEJO SUPERIOR DE INVESTIGACIONES CIENTIFICAS Y ES DE ACCESO LIBRE. CREATIVE COMMONS LICENSE 4.O BY 


\begin{tabular}{llllcl} 
P3A & Gr.1 $(\mathrm{N}=14)$ & 3 años & 14 & 8 & 6 \\
P3B & Gr.2 $(\mathrm{N}=13)$ & 3 años & 13 & 5 & 8 \\
P4A & Gr.3 $(\mathrm{N}=11) \mathrm{Gr} .4(\mathrm{~N}=10)$ & 4 años & 21 & 12 & 9 \\
P4B & Gr.5 $(\mathrm{N}=10) \mathrm{Gr} .6(\mathrm{~N}=10)$ & 4 años & 20 & 7 & 13 \\
P5A & Gr.7 $(\mathrm{N}=10) \mathrm{Gr} .8(\mathrm{~N}=9)$ & 5 años & 19 & 10 & 9 \\
P5B & Gr.9 $(\mathrm{N}=9)$ Gr.10 $(\mathrm{N}=8)$ & 5 años & 17 & 6 & 11 \\
\hline
\end{tabular}

Fuente: elaboración propia

En la Tabla 1, se observa la configuración de los grupos clase naturales de Educación Infantil del centro, a partir de los cuales se crearon 10 subgrupos desdoblando los grupos más numerosos (P4 y P5). En cuanto al género, se buscó mantener la composición con el equilibrio original. Finalmente, también se tuvo en consideración para su distribución el reparto de estudiantes con necesidades educativas especiales.

\subsection{Diseño del espacio}

La ambientación sonora del espacio, en el aula de música del centro, se diseñó con las propuestas que se nombran y describen en la Figura1, en la que se reproduce el enunciado con el que se presentó al alumnado.

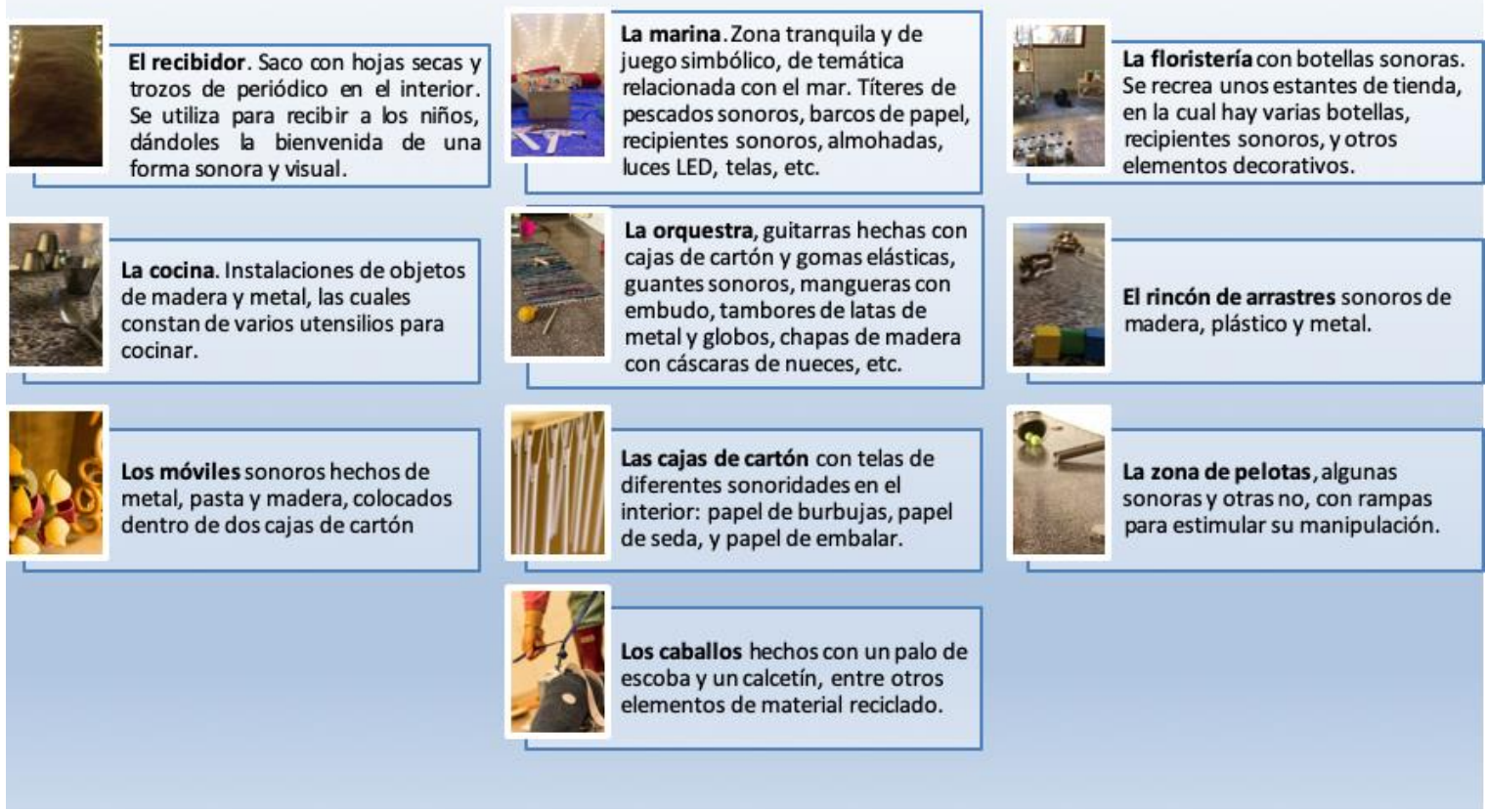

Figura 1. Elementos instalados en el espacio como ambiente de exploración sonora

En el diseño y elaboración del material participaron un grupo de colaboradores formado por las seis tutoras de etapa y el maestro de música del centro. Estos docentes aportaron propuestas

@MARTA CIURANA GONZÁLEZ Y MIQUEL ALSINA TARRÉS. THE CONTENT OF THIS ARTICLE IS THE SOLE RESPONSIBILITY OF THE AUTHORS. THE REVISTA ELECTRÓNICA DE LEEME AND UNIVERSITAT DE VALĖNCIA ARE NOT LIABLE FOR ANY LEGAL ACTIONS THAT MAY ARISE INVOLVING THE ARTICLE'S CONTENT, REVISTA ELECTRÓNICA DE LEEME -LISTA ELECTRÓNICA EUROPEA DE MÚSICA EN LA EDUCACIÓN-HTTP://OJS.UV.ES/INDEX/PHP/LEEME/INDEX. ISSN: 1575-9563. EDITORES: UNIVERSIDAD DE VALENCIA Y JESÚS TEJADA GIMÉNEZ. VISIBILIDAD DE ESTA REVISTA: SCOPUS, EMERGING SOURCES CITATION INDEX (CLARIVATE), EBSCO, CINDOC (CSIC), CITEFACTOR, COPAC, DIALNET, DICE (CSIC), DOAJ, E-REVISTAS (CSIC), EBSCO PREMIER, ERIH+, GALE CENGAGE LEARNING, IN-RECS, IRESIE, LATINDEX, MIAR, OCLC WORLDCAT, RESH, REDIB, RILM CORE JOURNALS, SUDOC, ULRICHS, ESTA REVISTA ESTÁ PUBLICADA CON EL APOYO INSTITUCIONAL DE REDIRIS-CONSEJO SUPERIOR DE INVESTIGACIONES CIENTIFICAS Y ES DE ACCESO LIBRE. CREATIVE COMMONS LICENSE 4.O BY 


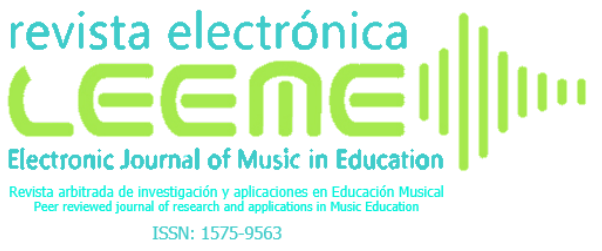

SECCIÓN ARTICULOS: MARTA CIURANA GONZÁLEZ Y MIQUEL ALSINA TARRÉS JUEGO Y SONIDO EN EDUCACIÓN INFANTIL: EL AULA DE MÚSICA COMO ESPACIO SONORO DE (REVISTA ELECTRÓNICA DE LEEME) NÚMERO 44, PP. $42-62$ HTTPS://OJS.UVIES/INDEX.PHP/LEEME/INDEX

ISSN: 1575-9563

de temas y centros de interés relacionados con el desarrollo curricular de sus programaciones anuales, así como materiales de todo tipo para la elaboración de los objetos, siguiendo criterios de sostenibilidad (reciclaje) y de seguridad.

La distribución del material en el aula, creando centros temáticos, se decidió dando prioridad a la ocupación homogénea del espacio y a su inclusión en el campo de visual de las dos cámaras instaladas para la grabación de las sesiones. En el resultado final se buscó conseguir un espacio ambientado coherentemente en su globalidad, organizado en diez puntos de actividad que resultaran atractivos y claramente diferenciados entre si. Se apostó por una disposición espacial en forma circular para un desplazamiento óptimo del alumnado, dejando espacio libre en el centro. De este modo, se consiguió una diferenciación clara de los espacios, donde el estudiantado podía identificar e interpretar fácilmente cada propuesta de forma autónoma. Las propuestas se presentaron, pues, como un conjunto armónico, desde una visión global del espacio sonoro, compuesto a su vez por pequeños ambientes (Capdevila, 2011).

\section{Resultados}

Los resultados que se exponen a continuación se agrupan en las cuatro categorías presentadas en la exposición del método, que se mantienen aquí como marco estructural e hipótesis de trabajo para el desarrollo del análisi. En consonancia con el paradigma exploratorio de la investigación, la codificación para cada categoría se ha desplegado a partir de una lista provisional, que fue revisada y reelaborada una vez hecha la recogida de datos cualitativos de la primera fase (piloto). Como explica Saldana (2016), los códigos provisionales deben tener una fuerte vinculación con la revisión bibliográfica, el marco conceptual y las preguntas de la investigación; así como resultados de otras investigaciones, observaciones en estudios piloto, y el conocimiento o experiencias previas de los investigadores.

En los resultados, se incluyen datos cualitativos y cuantitativos. Los primeros, cualitativos, expresan las frecuencias de los registros cuantificandas con el peso de cada categoría en los distintos cursos de la etapa. Las codificiaciones, en cambio, se argumentan a partir de los datos cualitativos, a partir de un análisis descriptivo e interpretativo.

\subsection{Uso sonoro y/o musical}

La categoría referida al uso sonoro de los materiales presenta unos registros altos en cada grupo de edad, siendo el superior el del grupo de $\mathrm{P} 4(51,9 \%, 73,2 \%$ y $69,4 \%)$ y registros poco significativos en las frecuencias más bajas $(4,8 \%, 2,4 \%$ y $5,6 \%)$. Las observaciones anotadas nos indican que los discentes con baja actividad se centraron en explorar uno o dos materiales, dejando a un lado el resto. 


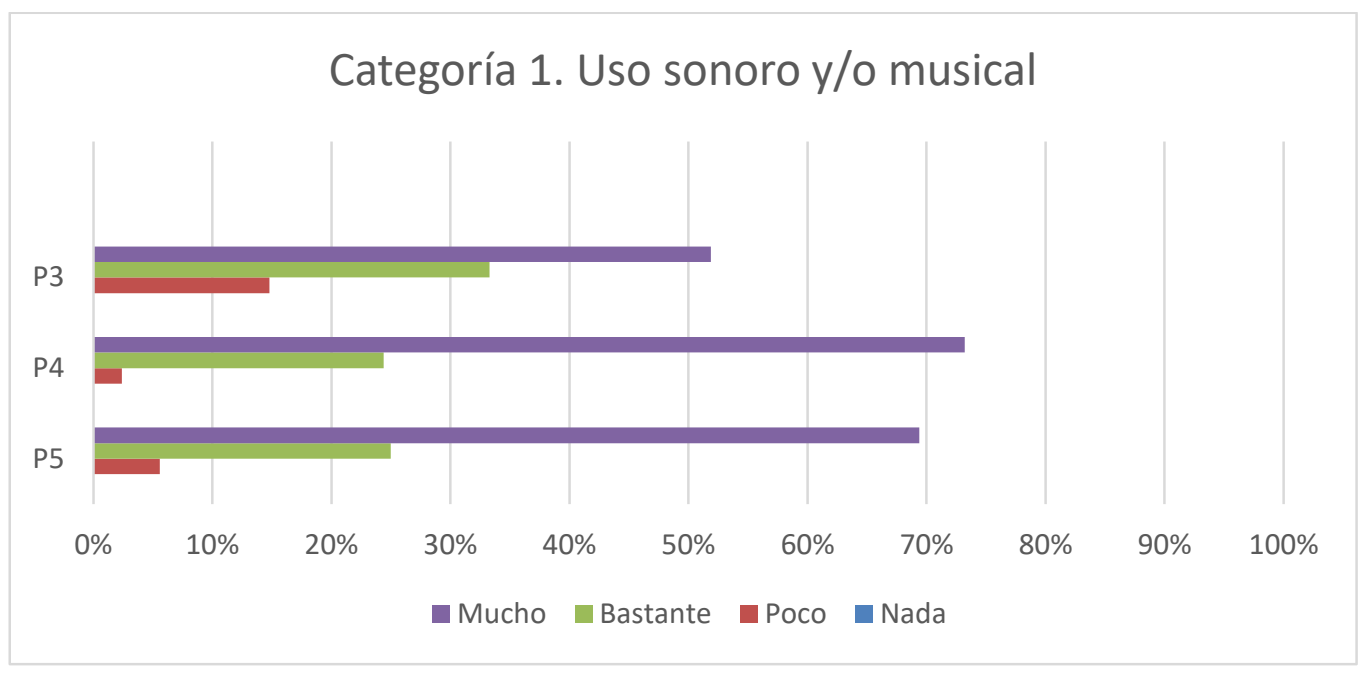

Figura 2. Gráfico de resultados en la categoría sobre el uso sonoro y/o musical

En relación con esta primera categoría, se han observado y codificado las siguientes acciones y aprendizajes:

- Exploración sonora. En un primer momento, la mayoría del alumnado ha realizado una exploración del objeto material que produce sonido. Se han interesado por el mecanismo de producción del sonido, haciendo variar los modos de poner en vibración el cuerpo sonoro. En un segundo momento, los educandos han realizado una exploración del objeto sonoro. Ha habido un cambio de atención del objeto material al objeto sonoro, factor que evoluciona con la edad y acelera el paso del paradigma físico al paradigma acústico.

- Clasificación sonora. Esta acción supone una capacidad avanzada y la realizaron espontáneamente una parte muy reducida de la muestra, una niña de P4 y dos niños de P5. Por otra parte, un grupo más numeroso de estudiantes en P4 y P5 clasificaron los objetos sonoros a partir de estrategias visuales, como la forma y contenido del objeto, por ejemplo, de una botella.

- Sonido - silencio. Entre los diferentes recipientes, había algunos de vacíos "sin sonido", aspecto que fue observado por parte del grupo de estudiantes. También, se han dado cuenta que algún objeto tenía materiales que, aunque se veían "no sonaban", por ejemplo, bolas de gelatina. A partir de objetos que de entrada "no suenan", en la muestra de P4 y P5 se han planteado problemas cognitivos recopilados en el registro cualitativo, del tipo “¿Por qué no suena?” “Cómo suena?” “¿Qué es lo que suena"?

- Fuerte - suave. El alumnado ha podido comprobar cómo hay objetos que suenan más fuerte o débil que otros, y ha podido tomar conciencia de los términos fuerte y suave, así como su diferenciación. De nuevo, el alumnado de P3, aunque lo ha

@MARTA CIURANA GONZÁLEZY Y MIQUEL ALSINA TARRÉS. THE CONTENT OF THIS ARTICLE IS THE SOLE RESPONSIBILITY OF THE AUTHORS. THE REVISTA ELECTRÓNICA DE LEEME AND UNIVERSITAT DE VALĖNCIA ARE NOT LIABLE FOR ANY LEGAL ACTIONS THAT MAY ARISE INVOLVING THE ARTICLE'S CONTENT. REVISTA ELECTRÓNICA DE LEEME -LISTA ELECTRÓNICA EUROPEA DE MÚSICA EN LA EDUCACIÓN-HTTP://OJS.UV.ES/INDEX/PHP/LEEME/INDEX. ISSN: 1575-9563. EDITORES: UNIVERSIDAD DE VALENCIA Y JESÚS TEJADA GIMÉNEZ. VISIBILIDAD DE ESTA REVISTA: SCOPUS, EMERGING SOURCES CITATION INDEX (CLARIVATE), EBSCO, CINDOC (CSIC), CITEFACTOR, COPAC, DIALNET, DICE (CSIC), DOAJ, E-REVISTAS ESTÁ PUBLICADA CON EL APOYO INSTITUCIONAL DE REDIRIS-CONSEJO SUPERIOR DE INVESTIGACIONES CIENTIFICAS Y ES DE ACCESO LIBRE. CREATIVE COMMONS LICENSE 4 .O BY 
podido experimentar en primera persona, no ha hecho ninguna manifestación verbal de esta cualidad.

- Exploración de instrumentos. Los educandos han explorado el sonido de diferentes instrumentos caseros. Han podido comprobar cómo cada uno de ellos hace un sonido diferente. Los instrumentos caseros en los que el alumnado ha dedicado más atención han sido: tambores, guitarras, embudos, y guantes sonoros.

- Relación canción - instrumento. Un grupo de niñas de P4 han utilizado las guitarras para acompañar una canción. En un primer momento, estaban tocando las guitarras de forma exploratoria y, acto seguido, una de las niñas ha comenzado a cantar una canción acompañándose con los sonidos de la guitarra. Se observa cómo su producción tenía intencionalidad, buscando expresión, comunicación e interacción entre ellas.

- Relación objeto sonoro - canción. Algunos estudiantes de todos los grupos han utilizado objetos, como los títeres en forma de pez, para cantar una determinada canción relacionada con el objeto. Han combinado el movimiento del títere con la voz cantada. Es decir, han asociado un objeto con una canción y con un timbre de voz.

- Discriminación de materiales a partir del sonido. En varias ocasiones, los educandos de P4 y P5, han acudido a los adultos para explicarles qué era lo que sonaba en los objetos sonoros. Han mostrado interés en descubrir qué material había dentro del objeto a partir de su sonido.

\subsection{Relación entre participantes}

El alumnado de P3 obtuvo un coeficiente muy bajo de interrelación, dato que aparece tanto en los registros de imágenes (77,8\% en el nivel "nada") como en el cuaderno de campo. En cambio, este factor aumenta notablemente en alumnado de P4 $(65,9 \%)$ y P5 $(66,7 \%)$.

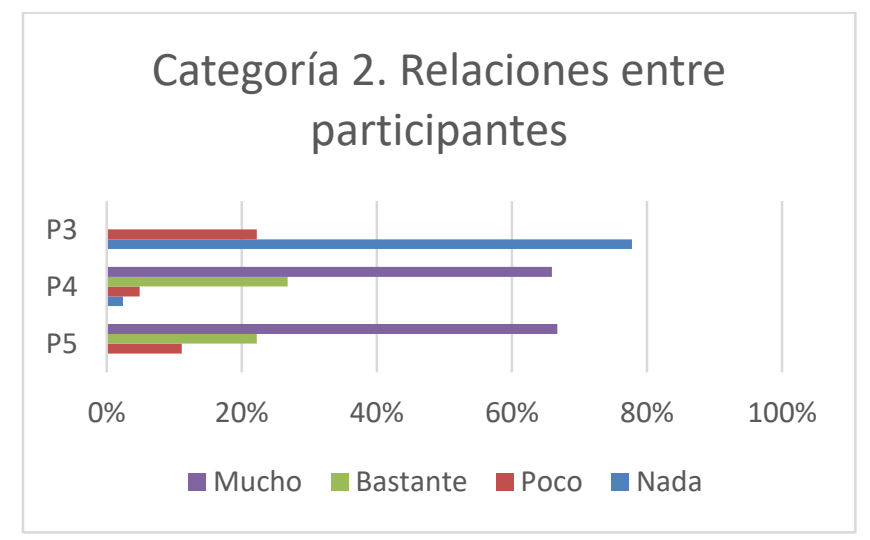

Figura 3. Gráfico sobre la categoría de interrelación entre participantes

@MARTA CIURANA GONZÁLEZ Y MIQUEL ALSINA TARRÉS. THE CONTENT OF THIS ARTICLE IS THE SOLE RESPONSIBILITY OF THE AUTHORS. THE REVISTA ELECTRÓNICA DE LEEME AND UNIVERSITAT DE VALĖNCIA ARE NOT LIABLE FOR ANY LEGAL ACTIONS THAT MAY ARISE INVOLVING THE ARTICLE'S CONTENT. REVISTA ELECTRÓNICA DE LEEME -LISTA ELECTRÓNICA EUROPEA DE MÚSICA EN LA EDUCACIÓN-HTTP://OJS.UV.ES/INDEX/PHP/LEEME/INDEX. ISSN: 1575-9563. EDITORES: UNIVERSIDAD DE VALENCIA Y JESÚS TEJADA GIMÉNEZ. VISIBILIDAD DE ESTA REVISTA: SCOPUS, EMERGING SOURCES CITATION INDEX (CLARIVATE), EBSCO, CINDOC (CSIC), CITEFACTOR, COPAC, DIALNET, DICE (CSIC), DOAJ, E-REVISTAS (CSIC), EBSCO PREMIER, ERIH+, GALE CENGAGE LEARNING, IN-RECS, IRESIE, LATINDEX, MIAR, OCLC WORLDCAT, RESH, REDIB, RILM CORE JOURNALS, SUDOC, ULRICHS, ESTA REVISTA
ESTA PUBLICADA CON EL APOYO INSTITUCIONAL DE REDIRIS-CONSEJO SUPERIOR DE INVESTIGACIONES CIENTIFICAS Y ES DE ACCESO LIBRE. CREATIVE COMMONS LICENSE 4.0 BY 
A continuación, se desglosa esta categoría a través de datos cualitativos, en la observación y el análisis de los siguientes códigos:

- Relaciones sociales e intercambios comunicativos. Se ha observado como el espacio de juego y exploración ha favorecido las relaciones sociales entre estudiantes, interaccionado entre ellos a través del juego libre. Sin embargo, esta interacción ha variado según el grupo de edad. En el alumnado de P3, ha predominado claramente un juego egocéntrico en solitario. Se trata de un juego en paralelo, no socializado. El único momento en el que se ha detectado interacción en la muestra de 3 años ha sido en el juego con embudos. En cuanto al grupo de estudiantes de P4, la mayoría han interaccionado entre ellos, agrupados en parejas o en grupos de 3 o 4 miembros. Por último, el alumnado de P5 se ha relacionado mucho entre sí, superando los registros de $\mathrm{P} 4$ y manteniendo la progresión creciente.

- Lenguaje verbal. con respecto al lenguaje verbal, este es inexistente en los registros de P3. Efectivamente, en los grupos de estudiantes de 3 años, se observan miradas, gestos y risas propias del lenguaje no verbal. En cambio, en el alumnado de P4 y P5 sí se verbalizan interacciones de forma complementaria al lenguaje no verbal, que sigue presente. En más de una ocasión, el estudiantado propuso ideas y acciones, con expresiones como: "vamos alli", "pruébalo tú", "hacemos esto", "déjame a mí", etc.

\subsection{Interacción con el material}

En esta categoría, se puede apreciar cómo en todos los grupos de edad no hay ningún alumno que no haya interaccionado con el material, en mayor o menor medida. La mayoría del alumnado ha interaccionado "mucho" (entre el 60\% y el 80\%) o "bastante"; aquellos que han interaccionado "poco" con el material $(14,8 \%, 2,4 \%$ y $5,6 \%)$, se han centrado todo el tiempo en explorar uno o dos materiales, dejando de relacionarse así con el resto.

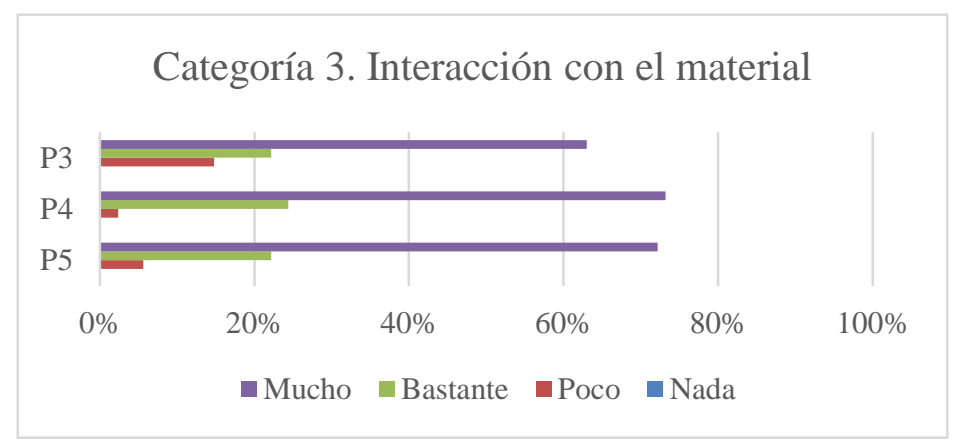

Figura 4. Gráfico sobre la categoría de interacción con el material

@MARTA CIURANA GONZÁLEZ Y MIQUEL ALSINA TARRÉS. THE CONTENT OF THIS ARTICLE IS THE SOLE RESPONSIBILITY OF THE AUTHORS. THE REVISTA ELECTRÓNICA DE LEEME AND UNIVERSITAT DE VALĖNCIA ARE NOT LIABLE FOR ANY LEGAL ACTIONS THAT MAY ARISE INVOLVING THE ARTICLE'S CONTENT. REVISTA ELECTRÓNICA DE LEEME -LISTA ELECTRÓNICA EUROPEA DE MÚSICA EN LA EDUCACIÓN-HTTP://OJS.UV.ES/INDEX/PHP/LEEME/INDEX. ISSN: 1575-9563. EDITORES: UNIVERSIDAD DE VALENCIA Y JESÚS TEJADA GIMÉNEZ. VISIBILIDAD DE ESTA REVISTA: SCOPUS, EMERGING SOURCES CITATION INDEX (CLARIVATE), EBSCO, CINDOC (CSIC), CITEFACTOR, COPAC, DIALNET, DICE (CSIC), DOAJ, E-REVISTAS (CSIC), EBSCO PREMIER, ERIH+, GALE CENGAGE LEARNING, IN-RECS, IRESIE, LATINDEX, MIAR, OCLC WORLDCAT, RESH, REDIB, RILM CORE JOURNALS, SUDOC, ULRICHS, ESTA REVIS
ESTÁ PUBLICADA CON EL APOYO INSTITUCIONAL DE REDIRIS-CONSEJO SUPERIOR DE INVESTIGACIONES CIENTIFICAS Y ES DE ACCESO LIBRE. CREATIVE COMMONS LICENSE 4.0 BY 
En relación con esta categoría, hemos interpretado los siguientes códigos de acción e interacción:

- Acciones matemáticas (clasificación y agrupación). Un grupo de discentes de P4 y P5 ha hecho una clasificación cualitativa de las botellas sonoras según los elementos en su interior. Por otra parte, uno de los alumnos de $\mathrm{P} 4$ ha agrupado dentro de una caja de cartón las botellas que ha ido encontrando en el espacio.

- Presencia de diferentes tipos de juego (funcional, simbólico, y de construcción). Por un lado, en el grupo de P3 ha predominado el juego funcional, en el que lo realmente importante son las acciones sobre los objetos, permitiendo así que el niño ejercite sus capacidades sensoriales y motrices. Por otra parte, en el alumnado de P4 y P5 ha predominado sobre todo el juego simbólico y, en menor medida, el juego de construcción. En cuanto al juego simbólico, se han producido situaciones de simulación: crear un pastel de cumpleaños, simular acciones de cocina, regar plantas, crear un río con papel de burbujas, barrer, espirar, cabalgar con los caballos, nadar como peces, disfrazarse como un preso, etc. En relación con el juego de construcción, este ha predominado entre el estudiantado de P5 y, en menor medida entre la muestra de P4. La construcción de torres, por ejemplo, ha sido siempre una acción individual, no colectiva.

- Necesidad de movimiento. Todos los grupos de edad han mostrado necesidad constante de movimiento, hecho que en ocasiones ha llevado a transformar el espacio al final de la sesión. Se observado cómo educandos de P3 y P4 han transformado el espacio de forma más global. En cambio, los de P5 han ido jugando en las diferentes zonas de manera más ordenada y han mantenido las zonas de juego iniciales establecidas por el adulto de manera más parecida. Principalmente en P4 y P5, se ha evidenciado mucha necesidad de guardar elementos dentro de las cajas y transportarlas, desplazando así los objetos por el espacio.

- Placer en los momentos de intimidad. Los registros muestran que diversos estudiantes de P4 como P5 han tenido tendencia a esconderse en algún momento de la sesión, buscando las zonas más oscuras e íntimas del espacio, así como la necesidad de vivir momentos de más calma. Por ejemplo, un grupo de discentes ha imaginado la presencia de un monstruo, y con la ayuda de las velas se ha escondido en la zona más oscura del pasillo. También un grupo ha estado cantando y tocando las guitarras en la zona más apartada del espacio. En cambio, los de P3 no han mostrado en ningún momento esta tendencia.

- Aparición de interrogantes cognitivos. El espacio y los objetos que lo configuran han propiciado interrogantes cognitivos al alumnado favoreciendo así los procesos cognitivos. Los datos, en las observaciones individuales, indican una presencia

@MARTA CIURANA GONZÁLEZY MIQUEL ALSINA TARRÉS. THE CONTENT OF THIS ARTICLE IS THE SOLE RESPONSIBILITY OF THE AUTHORS. THE REVISTA ELECTRÓNICA DE LEEME AND UNIVERSITAT DE VAL ĖNCIA ARE NOT LIABLE FOR ANY LEGAL ACTIONS THAT MAY ARISE INVOLVING THE ARTICLE'S CONTENT. REVISTA ELECTRÓNICA DE LEEME —LISTA ELECTRÓNICA EUROPEA DE MÚSICA EN LA EDUCACIÓN-HTTP://OJS.UV.ES/INDEX/PHP/LEEME/INDEX. ISSN: 1575-9563. EDITORES: UNIVERSIDAD DE VALENCIA Y JESÚS TEJADA GIMÉNEZ. VISIBILIDAD DE ESTA REVISTA: SCOPUS, EMERGING SOURCES CITATION INDEX (CLARIVATE), EBSCO, CINDOC (CSIC), CITEFACTOR, COPAC, DIALNET, DICE (CSIC), DOAJ, E-REVISTAS (CSIC), EBSCO PREMIER, ERIH+, GALE CENGAGE LEARNING, IN-RECS, IRESIE, LATINDEX, MIAR, OCLC WORLDCAT, RESH, REDIB, RILM CORE JOURNALS, SUDOC, ULRICHS, ESTA REVISTA ESTÁ PUBLICADA CON EL APOYO INSTITUCIONAL DE REDIRIS-CONSEJO SUPERIOR DE INVESTIGACIONES CIENTIFICAS Y ES DE ACCESO LIBRE. CREATIVE COMMONS LICENSE 4.O BY 
mayoritaria de interrogantes relacionados con el origen y naturaleza del sonido: ¿Qué suena?, ¿Por qué suena?, ¿Cómo suena? Y en una fase más avanzada, a su ordenación: ¿Cómo puedo ordenar los sonidos?, ¿Qué sonidos son iguales?, ¿Cuáles son diferentes o parecidos?, etc.

- Exploración sensorial. Al tratarse de un espacio multisensorial, el alumnado ha aprovechado para explorar su gran abanico de posibilidades sensoriales a través de los todos los sentidos, potenciando especialmente tacto, vista y oído.

- Pensamiento creativo y divergente. En este espacio, el alumnado ha encontrado un camino para poder desarrollar todo lo que está depositado en su imaginario. El hecho de ofrecer objetos con múltiples usos ha permitido que el educando muestre su personalidad creativa y divergente, yendo mucho más allá de lo que se espera en un primer momento por parte del adulto. También, se ha detectado cuales eran aquellos estudiantes más inseguros o que necesitaban de la interacción con el adulto de manera más constante. Por ejemplo, en el registro de observaciones en un grupo de P4, se recoge cómo un caso con ciertas dificultades madurativas buscaba repetidamente la aprobación del adulto para escoger el material y manipularlo.

\subsection{Interés y motivación}

Los resultados, en este apartado, nos muestran cómo la propuesta ha generado una muy buena respuesta en el alumnado de todos los grupos de edad, ya que en su mayoría han mostrado "mucho" interés en la actividad $(92,6 \%, 97,6 \%, 97,2 \%)$. Los pocos estudiantes que han mostrado "poco" interés en la propuesta $(3,7 \%$ y $2,8 \%)$, participaron en la sesión con problemas de relación previos o responde a diagnósticos previos que dificultan su progreso e integración en el grupo.

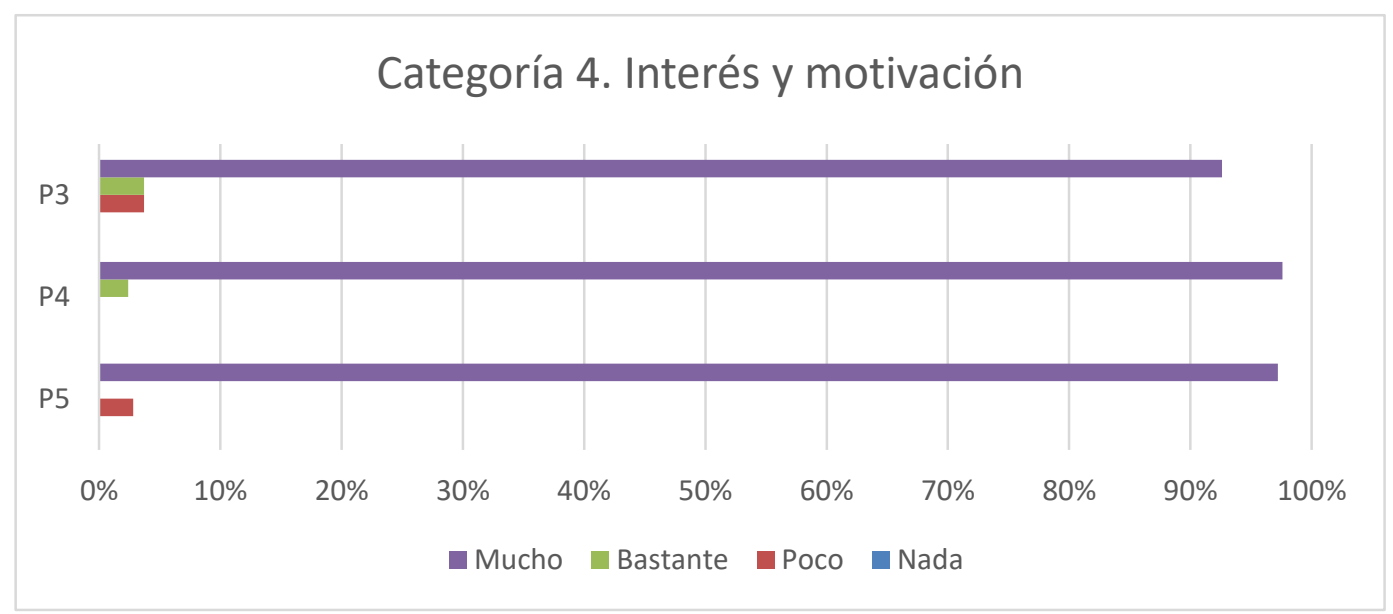

Figura 5. Gráfico de la categoría de interés y motivación

@MARTA CIURANA GONZÁLEZ Y MIQUEL ALSINA TARRÉS. THE CONTENT OF THIS ARTICLE IS THE SOLE RESPONSIBILITY OF THE AUTHORS. THE REVISTA ELECTRÓNICA DE LEEME AND UNIVERSITAT DE VALĖNCIA ARE NOT LIABLE FOR ANY LEGAL ACTIONS THAT MAY ARISE INVOLVING THE ARTICLE'S CONTENT. REVISTA ELECTRÓNICA DE LEEME -LISTA ELECTRÓNICA EUROPEA DE MÚSICA EN LA EDUCACIÓN-HTTP://OJS.UV.ES/INDEX/PHP/LEEME/INDEX. ISSN: 1575-9563. EDITORES: UNIVERSIDAD DE VALENCIA Y JESÚS TEJADA GIMÉNEZ. VISIBILIDAD DE ESTA REVISTA: SCOPUS, EMERGING SOURCES CITATION INDEX (CLARIVATE), EBSCO, CINDOC (CSIC), CITEFACTOR, COPAC, DIALNET, DICE (CSIC), DOAJ, E-REVISTAS
(CSIC), EBSCO PREMIER, ERIH+, GALE CENGAGE LEARNING, IN-RECS, IRESIE, LATINDEX, MIAR, OCLC WORLDCAT, RESH, REDIB, RILM CORE JOURNALS, SUDOC, ULRICHS, ESTA REVISTA ESTÁ PUBLICADA CON EL APOYO INSTITUCIONAL DE REDIRIS-CONSEJO SUPERIOR DE INVESTIGACIONES CIENTIFICAS Y ES DE ACCESO LIBRE. CREATIVE COMMONS LICENSE 4.O BY 
- Exteriorización de emociones, sentimientos y actitudes. A lo largo de las sesiones, se han puesto en juego muchas emociones que han sido exteriorizadas por el educando. Han predominado las emociones que se han etiquetado como positivas (sorpresa, alegría, incertidumbre, bienestar, etc.). Sin embargo, también han surgido, con menor grado, sentimientos de enojo con algún compañero o frustración ante las normas de la actividad. Estos momentos de conflicto también han permitido introducir la gestión de las emociones y encontrar soluciones compartidas.

Para concluir este capítulo, en la Figura 6, se recogen todas las acciones de interacción y aprendizaje observados en un formato visual y esquemático.
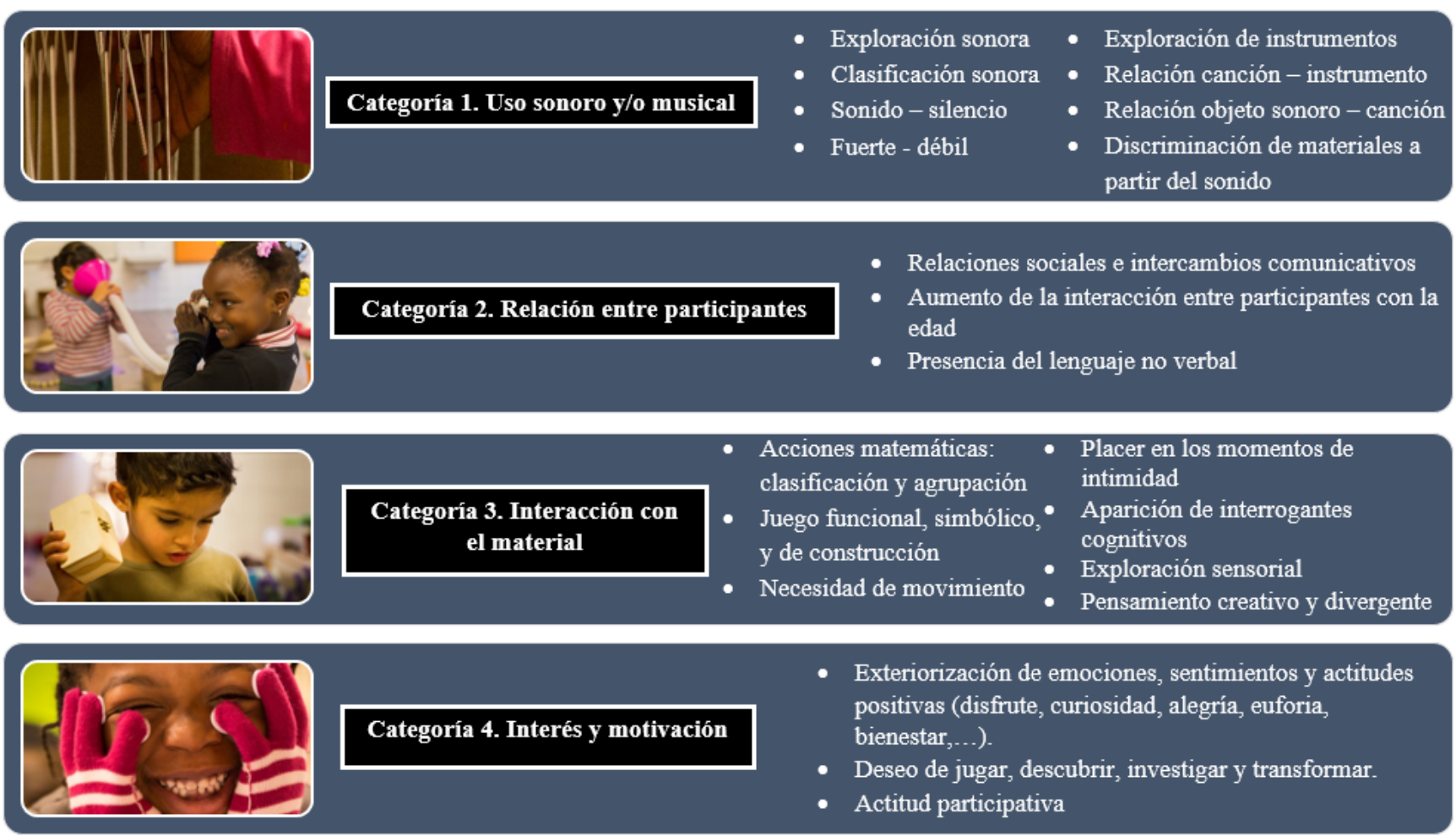

Figura 6. Cuadro sintético de las acciones y aprendizajes codificados en cada categoría de análisis

\section{Discusión de resultados y conclusiones}

Los resultados que se acaban de exponer responden a las preguntas de la investigación con suficiente detalle y diversos niveles de argumentación. En la primera pregunta, nos preguntábamos cómo interaccionaría el alumnado de Infantil en un medio dispuesto para la exploración sonora. Si para Iglesias (2008) las actividades a desarrollar en un ambiente de

@MARTA CIURANA GONZÁLEZ Y MIQUEL ALSINA TARRÉS. THE CONTENT OF THIS ARTICLE IS THE SOLE RESPONSIBILITY OF THE AUTHORS. THE REVISTA ELECTRÓNICA DE LEEME AND UNIVERSITAT DE VALĖNCIA ARE NOT LABLE FOR ANY LEGAL ACTIONS THAT MAY ARISE INVOLVING THE ARTICLE'S CONTENT. REVISTA ELECTRÓNICA DE LEEME —LISTA ELECTRÓNICA EUROPEA DE MÚSICA EN LA EDUCACIÓN-HTTP://OJS.UV.ES/INDEX/PHP/LEEME/INDEX. ISSN: 1575-9563. EDITORES: UNIVERSIDAD DE VALENCIA Y JESÚS TEJADA GIMÉNEZ. VISIBILIDAD DE ESTA REVISTA: SCOPUS, EMERGING SOURCES CITATION INDEX (CLARIVATE), EBSCO, CINDOC (CSIC), CITEFACTOR, COPAC, DIALNET, DICE (CSIC), DOAJ, E-REVISTAS (CSIC), EBSCO PREMIER, ERIH+, GALE CENGAGE LEARNING, IN-RECS, IRESIE, LATINDEX, MIAR, OCLC WORLDCAT, RESH, REDIB, RILM CORE JOURNALS, SUDOC, ULRICHS, ESTA REVISTA ESTÁ PUBLICADA CON EL APOYO INSTITUCIONAL DE REDIRIS-CONSEJO SUPERIOR DE INVESTIGACIONES CIENTIFICAS Y ES DE ACCESO LIBRE. CREATIVE COMMONS LICENSE 4.O BY 
aprendizaje pueden responder a hasta doce tipologías diferentes, en nuestro caso observamos correspondencia con seis del listado de la autora, quien ya advierte que su taxonomía debe adaptarse a cada contexto y experiencia. Es nuestra investigación las tipologías de actividad han sido:

- Actividades de encuentro y comunicación.

- Actividades de juego simbólico y juego libre.

- Actividades de movimiento y expresión corporal.

- Actividades de manipulación y experimentación.

- Actividades con juegos didácticos estructurados y no estructurados.

- Actividades disruptivas.

Así mismo, el proyecto de ambiente de aprendizaje sonoro ha transformado el concepto de espacio, convirtiendo el aula de música en un lugar singular en experiencias y aprendizajes. En este sentido, los resultados de la investigación reafirman el potencial pedagógico y la estrecha relación entre proyecto educativo y uso del espacio escolar. El factor físico, material y ambiental, condicionan y definen la propuesta didáctica en sus objetivos y desarrollo, siendo el concepto de ambientes de aprendizaje una propuesta muy adecuada para "multiplicar las posibilidades de exploración y acción del niño y propiciar múltiples relaciones que favorecen la creación de una comunidad de convivencia y aprendizaje" (Riera, Ferrer y Ribas, 2014, p.32).

Más aún, en el contexto particular en el que se ha desarrollado nuestra investigación, marcado por un fuerte componente de exclusión social, se pone de relieve el valor de la experiencia sonoro musical como herramienta inclusiva en prácticas que son transformadoras por su valor de socialización y empoderamiento. En sintonía, por ejemplo, con la investigación de Balongo y Mérida (2016), donde se exponen resultados positivos de inclusión en un estudio de caso que combina el clima del aula con los proyectos de trabajo y los ambientes de aprendizaje. Inclusión y trasformación son cualidades que reclaman protagonismo en nuestras conclusiones puesto que, como defiende Bonàs (2010), todo concepto no predeterminado sobre el uso del espacio promueve la iniciativa, la creatividad y un pensamiento abierto.

Eslava (2016) ha planteado un diálogo continuo y necesario entre la arquitectura y la pedagogía. Argumentando la necesidad de ofrecer al alumnado entornos que estimulen su desarrollo lúdico. Por eso mismo, el autor habla del beneficio de espacios transformables e instalaciones efímeras. Nuestros resultados concuerdan, efectivamente, con ese componente estimulante y lo enmarcan, de forma coherente, con el desarrollo curricular vigente. Estímulos materiales y ambientales que han propiciado la aparición de aprendizajes diversos, convirtiéndose así en un recurso educativo rico, transversal y completo en si mismo.

Por otro lado, desde una óptica disciplinar, en los resultados expuestos se demuestra cómo, a partir de una intervención didáctica centrada en la sensibilización musical, se favorecen 


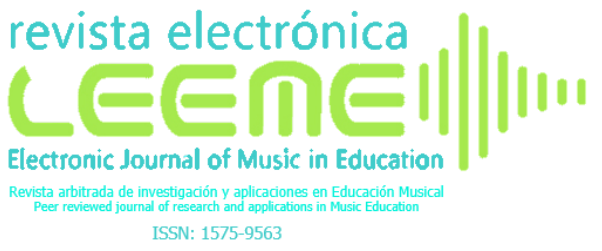

SECCIÓN ARTICULOS: MARTA CIURANA GONZÁLEZ Y MIQUEL ALSINA TARRÉS JUEGO Y SONIDO EN EDUCACIÓN INFANTIL: EL AULA DE MÚSICA COMO ESPACIO SONORO DE (REVISTA ELECTRÓNICA DE LEEME) NÚMERO 44, PP. 42-62 HTTPS://OJS.UV.ES/INDEX.PHP/LEEME/INDEX DOI: $10.7203 /$ LEME 44.15595

las relaciones sociales, la exteriorización de emociones, el desarrollo del pensamiento creativo y divergente, la aparición de nuevos interrogantes cognitivos, entre otros beneficios.

La segunda pregunta de la investigación se refería a la evolución observable a lo largo los tres niveles de la etapa. Los datos analizados determinan, efectivamente, diferencias notables en las conductas del alumnado del grupo de edad, principalmente en referencia al tipo de juego, las relaciones sociales, y los intercambios comunicativos. Los datos recogidos avalan algunos de los referentes de nuestro marco teórico, en relación con el proceso madurativo y desarrollo de capacidades de interacción con el mundo sonoro (Calvo y Bernal, 2000; Pascual, 2006), así como la evolución de sus gustos musicales. En este último aspecto, los resultados publicados por PérezMoreno y Reverté (2019) están centrados en una muestra de población de 4 años y aquí la ampliamos al contexto de toda la etapa educativa.

Ofreciendo al educando un espacio ambientado para el aprendizaje, rico en estímulos y posibilidades de acción, se incide en gran medida en el refuerzo de su autonomía. Se ha demostrado cómo un gran número de aprendizajes han sucedido espontáneamente a través del juego libre, como han defendido incansablemente autores como Tonucci (2017). Efectivamente, las observaciones confirman y refuerzan las enormes posibilidades del juego como estrategia educativa, que junto al estímulo de lo lúdico ofrece un entorno de bienestar donde los escolares se interrelacionan entre iguales. En palabras de Pedreira (2015, p.44): "supone la creación de un tiempo relajado para participar en el juego de los otros o concentrarse en el propio, para sentirse bien, para disfrutar de la situación y de la compañía”.

\section{Limitaciones y prospectiva}

Esta investigación tiene las limitaciones propias de todo enfoque exploratorio, en cuanto a la provisionalidad de los resultados y parcialidad en su interpretación. Sin embargo, se ponen a examen las cualidades y beneficios de un planteamiento metodológico mayoritariamente implantado en las aulas de Educación Infantil y, actualmente, también presente en algunos proyectos educativos de Primaria. Por esta razón, interesa indagar para reivindicar el papel de la educación musical en este paradigma metodológico y en una etapa educativa donde a menudo carece del protagonismo suficiente. El futuro inmediato requiere de más investigación e implicación desde la formación docente, para acabar incidiendo en una mejora, en cantidad y en calidad, de la presencia de la música en los proyectos educativos de la Educación Infantil.

\section{Referencias}

Abad, J. (2008). El placer y el displacer en el juego espontaneo infantil. Arteterapia, 3, 167188. Recuperado de: https://revistas.ucm.es/index.php/ARTE/ 
Abad, J. y Ruiz, A. (2014). Contexto de simbolización y juego: La propuesta de las instalaciones. Aula de Infantil, 77, 11-15. Recuperado de: https://www.grao.com/es/producto/contextos-de-simbolizacion-y-juego

Balongo, E. y Mérida, R. (2016). El clima de aula en los proyectos de trabajo. Crear ambientes de aprendizaje para incluir la diversidad infantil. Perfiles educativos, 38(152), 146-162. Recuperado de: http://www.iisue.unam.mx/perfiles/perfiles_articulo

Bernal, J., Calvo, Mª L. (2000). Didáctica de la música: la expresión musical en educación infantil. Málaga, España: Ediciones Aljibe.

Blaking, J. (1974) How musical is man? Washington, USA: University of Washington Press.

Bonàs, M. (2010). El espacio vacío. Tiempos y espacios de posibilidades. Aula de Innovación educativa, (193-194), 32-35. Recuperado de: http://caps.educacion.navarra.es/infantil/

Cabello, M.J. (2011). La organización del espacio en educación infantil: poderoso instrumento docente. Pedagogía Magna, 11, 196-203. Recuperado de: https://dialnet.unirioja.es/servlet/articulo?codigo $=3629187$

Capdevila, R. (2011). Com sona la meva escola? In-fàn-ci-a, 181, 7-12.

Creswell, J.W. y Creswell, J.D. (2018). Research Design: Qualitative, Quantitative, and Mixed Methods Approaches, (5 $\left.{ }^{\mathrm{a}} \mathrm{ed}.\right)$. London, UK: Sage.

Delalande, F. (1995). La música es un juego de niños. Buenos Aires, Argentina: Ricordi Americana S.A.E.C.

Delalande, F. y Cornara, S. (2010). Sound explorations from the ages of 10 to 37 months: the ontogenesis of musical conducts. Music Education Research, 12(3), 257-268. doi: $10.1080 / 14613808.2010 .504812$

Delalande, F. (2013). Las conductas musicales. Santander, España: Editorial Universidad de Cantabria.

Díez, M. (2013). 10 ideas claves. La educación infantil. Barcelona, España: Editorial Graó.

Eslava, C. (2016). Espais oblidats, entorns d'aprenentatge: codi de bones pràctiques per actuar en zones existents. Guix d'Infantil, 88, 11-15.

Gairín, J. (1995). El reto en la organización de los espacios. Aula de Innovación educativa, 35, 45-50. Recuperado de: https://ddd.uab.cat/record/183074

@MARTA CIURANA GONZÁLEZY MIQUEL ALSINA TARRÉS. THE CONTENT OF THIS ARTICLE IS THE SOLE RESPONSIBILITY OF THE AUTHORS. THE REVISTA ELECTRÓNICA DE LEEME AND UNIVERSITAT DE VALĖNCIA ARE NOT LIABLE FOR ANY LEGAL ACTIONS THAT MAY ARISE INVOLVING THE ARTICLE'S CONTENT REVISTA ELECTRÓNICA DE LEEME -LISTA ELECTRÓNICA EUROPEA DE MÚSICA EN LA EDUCACIÓN-HTTP://OJS.UV.ES/INDEX/PHP/LEEME/INDEX. ISSN: 1575-9563. EDITORES: UNIVERSIDAD DE VALENCIA Y JESÚS TEJADA GIMÉNEZ. VISIBILIDAD DE ESTA REVISTA: SCOPUS, EMERGING SOURCES CITATION INDEX (CLARIVATE), EBSCO, CINDOC (CSIC), CITEFACTOR, COPAC, DIALNET, DICE (CSIC), DOAJ, E-REVISTAS (CSIC), EBSCO PREMIER, ERIH+, GALE CENGAGE LEARNING, IN-RECS, IRESIE, LATINDEX, MIAR, OCLC WORLDCAT, RESH, REDIB, RILM CORE JOURNALS, SUDOC, ULRICHS, ESTA REVISTA ESTÁ PUBLICADA CON EL APOYO INSTITUCIONAL DE REDIRIS-CONSEJO SUPERIOR DE INVESTIGACIONES CIENTIFICAS Y ES DE ACCESO LIBRE. CREATIVE COMMONS LICENSE 4.O BY 
Generalitat de Catalunya (2016). Currículum i orientació. Educación infantil (Segon Cicle). Barcelona, España: Departament d'Ensenyament.

Hallam, S. (2010). The power of music: Its impact on the intellectual, social and personal development of children and young people. International Journal of Music Education, 28, 269-289. doi: 10.1177/0255761410370658.

Iglesias, M.L. (2008). Observación y evaluación del ambiente de aprendizaje en educación infantile: dimensiones y variables a considerar. Revista Iberoamericana de educación, (47), 49-70. doi: 10.35362/rie470704

Ilari, B. (2016). Music in the early years: Pathways into the social world. Research Studies in Music Education, 38(1), 23-39. Doi: 10.1177/1321103X16642631

Jäncke, L. (2009). Music drives brain plasticity. F1000 Biology Reports, 1(78), 1-7. doi: 10.3410/B1-78.

Kemple, K., Batey, J.J. y Hartle, L.C. (2004). Music play: Creating centers for musical play and exploration. Young Children, 59, 30-37. Recuperado de: https://cpin.us/sites/default/files/docs/music play Young Children.pdf

Laorden, C. y Pérez, C. (2002). El espacio como elemento facilitador del aprendizaje. Una experiencia en la formación inicial del profesorado. Pulso 25, 133-146. Recuperado de: https://dialnet.unirioja.es/servlet/articulo?codigo $=243780$

Leavy, P. (2017). Research design. Quantitative, Qualitative, Mixed Methods, Art-Based, and Community-Based Participatory Research Approaches. New York \& London: Guilford.

Levinowitz, L.M. (1998). The importance of music in early childhood. General Music Today, 12(1), 4-7. doi: $10.1177 / 104837139801200103$

Pascual, P. (2006). Didáctica de la música para educación infantil. Madrid, España: Pearson Educación.

Pedreira, M. (2015). Espais de joc. Guix d'Infantil, 80, 44-45. Disponible en: https://www.grao.com/es/producto/espais-de-joc

Peretz, I. (2006). The nature of music from a biological perspective. Cognition, 100(1), 1-32. doi: 10.1016/ j.cognition.2005.11.004.

Pérez-Moreno, J. y Reverté, L (2019). Las actividades musicales preferidas de la voz de los propios niños y niñas de cuatro años. Un estudio de caso. Revista Electrónica de LEEME, 43, 19-34. doi: 10.7203/LEEME43.13985

@MARTA CIURANA GONZÁLEZY MIQUEL ALSINA TARRÉS. THE CONTENT OF THIS ARTICLE IS THE SOLE RESPONSIBILITY OF THE AUTHORS. THE REVISTA ELECTRÓNICA DE LEEME AND UNIVERSITAT DE VALĖNCIA ARE NOT LABLE FOR ANY LEGAL ACTIONS THAT MAY ARISE INVOLVING THE ARTICLE'S CONTENT REVISTA ELECTRÓNICA DE LEEME -LISTA ELECTRÓNICA EUROPEA DE MÚSICA EN LA EDUCACIÓN-HTTP://OJS.UV.ES/INDEX/PHP/LEEME/INDEX, ISSN: 1575-9563. EDITORES: UNIVERSIDAD DE VALENCIA Y JESÚS TEJADA GIMÉNEZ. VISIBILIDAD DE ESTA REVISTA: SCOPUS, EMERGING SOURCES CITATION INDEX (CLARIVATE), EBSCO, CINDOC (CSIC), CITEFACTOR, COPAC, DIALNET, DICE (CSIC), DOAJ, E-REVISTAS (CSIC), EBSCO PREMIER, ERIH+, GALE CENGAGE LEARNING, IN-RECS, IRESIE, LATINDEX, MIAR, OCLC WORLDCAT, RESH, REDIB, RILM CORE JOURNALS, SUDOC, ULRICHS, ESTA REVISTA ESTÁ PUBLICADA CON EL APOYO INSTITUCIONAL DE REDIRIS-CONSEJO SUPERIOR DE INVESTIGACIONES CIENTIFICAS Y ES DE ACCESO LIBRE. CREATIVE COMMONS LICENSE 4.O BY 
Riera, C.M., Ferrer, C. y Ribas, C. (2014). La organización del espacio por ambientes de aprendizaje en la Educación Infantil: significados, antecedentes y reflexiones. Revista Latinoamericana de Educación Infantil, 3(2). 19-39. Recuperado de: http://www.usc.es/revistas/index.php/reladei/article/view/4726/5081

Río Sadornil, D. (2003) Métodos de investigación en educación. Vol. 1: Proceso y diseños no complejos. Madrid, España: UNED.

Romero, V. y Gómez, M. (2011). El juego infantil y su metodología. Barcelona, España: Editorial Altamar.

Saldana, J. (2016). The coding manual for qualitative researchers (3rd ed.). London, UK: SAGE.

Saenz-Rico, B., Benítez, L., Neira, J.M., Sobrino, M.R. y D’angelo, E. (2015). Perfiles profesionales de futuros maestros para el desarrollo sostenible desde un modelo formativo centrado en el diseño de ambientes de aprendizaje. Foro de Educación, 13(19), 141-163. doi: 10.14516/fde.2015.013.019.007

Silvente, J. (2017). Camins cap al disseny d'uns espais amables i habitables. Infància, 219,10-14.

Tammerman, N. (2000). An investigation of the music activity preferences of pre-school children. British Journal of Music Education, 17(1), 51-60. doi: 10.1017/ S0265051700000140.

Thompson, C. (2003). Kinderculture in the art classroom: Early childhood art and the mediation of culture. Studies in Art Education, 44(2), 135-146. doi: 10.1080/00393541.2003.11651734

Tonucci, F. (2017). Con ojos de maestro. Madrid, España: Losada.

Torelli, L. y Durrett, C. (1996). Landscape for Learning: The Impact of Classroom Design on Infants and Toddlers. Early Childhood News, 8, 12-17.

Trueba, B. (2015). Espacios en armonía: Propuestas de actuación en ambientes para la infancia (1a ed.). Barcelona, España: Octaedro - Rosa Sensat.

Young, S. (2003). Time-space structuring in spontaneous play on educational percussion instruments among three- and four-year-olds. British Journal of Music Education, 20(1), 45-59. doi: $10.1017 / \mathrm{S} 0265051702005284$

Young, S. (2016). Early childhood music education research: An overview. Research Studies in Music Education, 38(1), 9-21. Doi: 10.1177/1321103X16640106

@MARTA CIURANA GONZÁLEZ Y MIQUEL ALSINA TARRÉS. THE CONTENT OF THIS ARTICLE IS THE SOLE RESPONSIBILITY OF THE AUTHORS. THE REVISTA ELECTRÓNICA DE LEEME AND UNIVERSITAT DE VALÈNCIA ARE NOT LIABLE FOR ANY LEGAL ACTIONS THAT MAY ARISE INVOLVING THE ARTICLE'S CONTENT. REVISTA ELECTRÓNICA DE LEEME —LISTA ELECTRÓNICA EUROPEA DE MÚSICA EN LA EDUCACIÓN-HTTP://OJS.UV.ES/INDEX/PHP/LEEME/INDEX. ISSN: 1575-9563. EDITORES: UNIVERSIDAD DE VALENCIA Y JESÚS TEJADA GIMÉNEZ. VISIBILIDAD DE ESTA REVISTA: SCOPUS, EMERGING SOURCES CITATION INDEX (CLARIVATE), EBSCO, CINDOC (CSIC), CITEFACTOR, COPAC, DIALNET, DICE (CSIC), DOAJ, E-REVISTAS (CSIC), EBSCO PREMIER, ERIH+, GALE CENGAGE LEARNING, IN-RECS, IRESIE, LATINDEX, MIAR, OCLC WORLDCAT, RESH, REDIB, RILM CORE JOURNALS, SUDOC, ULRICHS, ESTA REVISTA ESTÁ PUBLICADA CON EL APOYO INSTITUCIONAL DE REDIRIS-CONSEJO SUPERIOR DE INVESTIGACIONES CIENTIFICAS Y ES DE ACCESO LIBRE. CREATIVE COMMONS LICENSE 4.O BY 
Young, S. y Gillen, J. (2007). Toward a Revised Understanding of Young Children's Musical Activities: Reflections from the "Day in the Life" Project. Current Musicology, 84, 7999. doi: 10.7916/D81N7ZR0

Zych, I., Ortega-Ruiz, R. y Sibaja, S. (2016). El juego infantil y el desarrollo afectivo: afecto, ajuste escolar y aprendizaje en la etapa preescolar. Infancia y Aprendizaje, 39(2), 380400. doi: $10.1080 / 02103702.2016 .1138718$

Zur, S. S. y Johnson-Green, E. (2008). Time to Transition: The Connection between Musical Free Play and School Readiness. Childhood Education, 84(5), 295-300. doi: $10.1080 / 00094056.2008 .10523029$ 